\title{
71 Année Fantastique Réflexivité et séries télévisées sur les chaînes de l'ORTF
}

\author{
Séverine Barthes \\ Université Sorbonne-Nouvelle / CEISME
}

On entend très régulièrement dire que les séries télévisées étrangères sont, et de loin, supérieures aux programmes français : après l'attention portée aux séries américaines à partir des années 1990, ce fut au tour du RoyaumeUni, d'Israël ou de la Suède d'être mis à l'honneur. Ce désamour des Français se traduit notamment dans le palmarès annuel des meilleures audiences, où la France est le seul pays dont la fiction nationale est battue par les séries américaines. Cependant, cette situation ne doit pas cacher le fait que ce changement est très récent et qu'il n'est pas seulement à mettre sur le fait que, le nombre de chaînes s'étant très rapidement multiplié depuis 1986, les écrans français ont été envahis par des fictions étrangères, moins chères que nos productions nationales ${ }^{1}$.

Il ne s'agit pas ici de se pencher plus avant sur les facteurs qui ont renversé, dans la réception des séries et des feuilletons, la hiérarchie entre productions nationales et productions étrangères, mais plutôt d'étudier à partir d'un cas précis — le fantastique au début des années 1970 l'inventivité des séries françaises avant la privatisation du $\mathrm{PAF}^{2}$. Ce genre est d'autant plus intéressant qu'il est aujourd'hui plutôt moribond dans les productions françaises actuelles. Si l'on s'intéresse à la production des séries fantastiques et de science-fiction en France, on note quelques périodes très productives (première moitié des années 1970, la décennie 1980 et le début des années 1990) et un nombre réduit le reste du temps (en particulier dans les années 2000, à l'exception d'un pic de trois programmes en 2007 ; voir figure 1$)^{3}$.

\footnotetext{
${ }^{1}$ Les faits contredisent le «mythe » de l’invasion de la télévision française par les séries américaines: les séries télévisées britanniques et américaines, par exemple, ont été un argument important pour attirer le public vers la $2^{\mathrm{e}}$ chaîne de l'ORTF à son lancement et pousser le public à acquérir de nouveaux téléviseurs pour la recevoir (Barthes 2012 : 127). Au début des années 1980, alors que tout l'audiovisuel français est encore public, on note même une égalité de volumes entre fictions françaises et fictions étrangères pour Antenne 2 (Barthes 2010 : 129).

${ }^{2}$ Rappelons que la première chaîne privée à péage, Canal Plus, date de 1984. Cependant, on retient généralement comma date-pivot 1986, année qui a vu la privatisation de TF1, jusqu'alors chaîne publique, et le début de l'exploitation commerciale des cinquième et sixième canaux hertziens (Michel 82-109).

${ }^{3}$ Notons que le fantastique s'est pleinement épanoui, à la télévision française, dans le genre du téléfilm, ce qui, d'un point de vue purement générique, déplace quelque peu les
} 


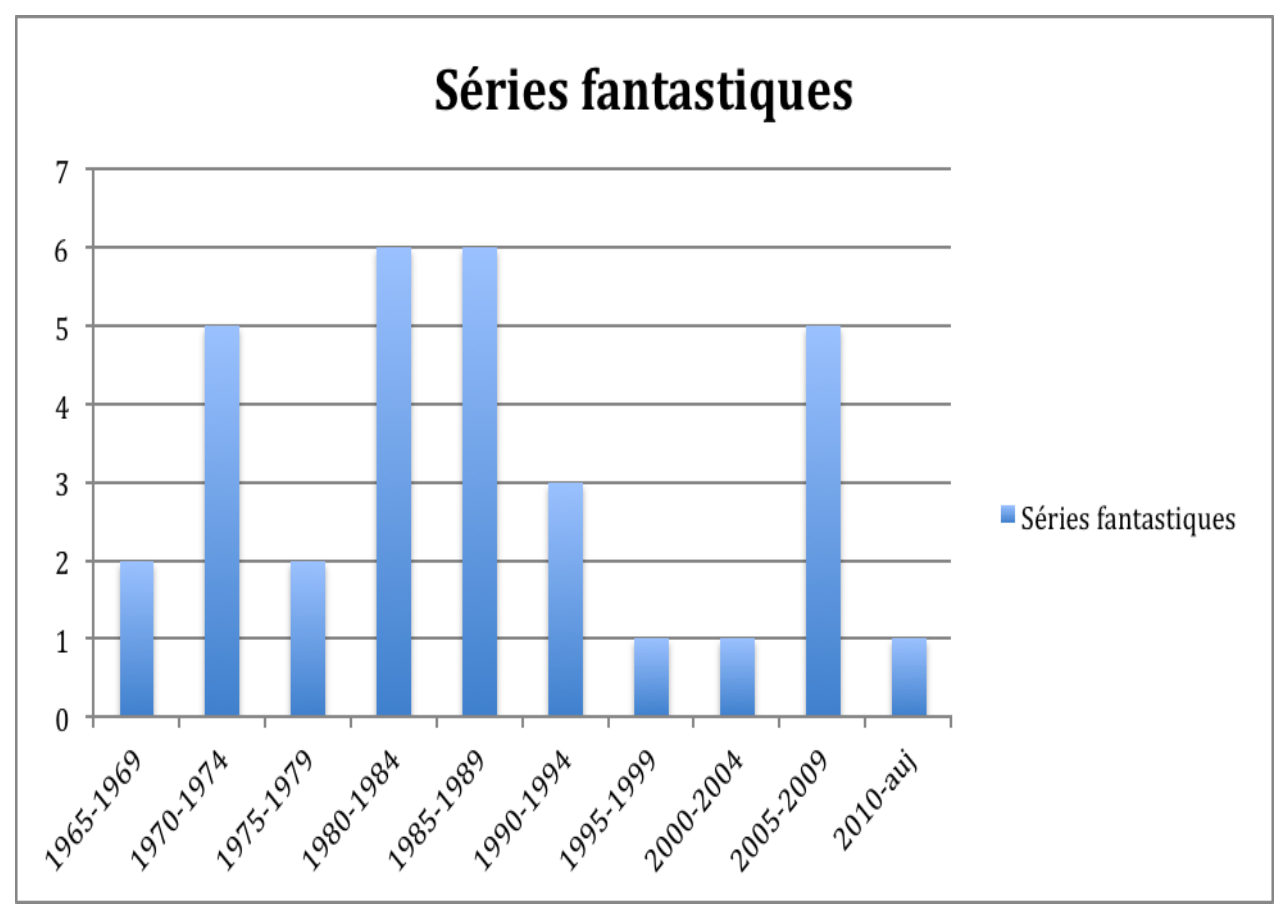

Fig. 1 : ventilation des séries fantastiques et de SF produites ou coproduites en France parpériode de cinq ans ${ }^{4}$

Si l'on se lance dans une comparaison entre série policière (souvent présenté comme le genre majeur de la fiction télévisée française) et série fantastique, on se rend compte que le déséquilibre est le résultat d'un processus qui a joué par assèchement des autres genres (voir figure 2).

enjeux et la distribution chronologique : "Entre les amateurs de "mauvais genres" et les réalisateurs libres d'adapter des textes de grands écrivains [...], le fantastique construit son nid à la télévision française. Baudou et Schléret situent d'ailleurs son plein essor entre 1962 et 1974. » (Lagane 47).

${ }^{4}$ Cette ventilation est fondée sur plusieurs recensements et listes de séries et feuilletons fantastiques et de SF, dont Wikipedia et l'ouvrage Merveilleux, Fantastique et Science-Fiction à la télévision française de Jacques Baudou et Jean-Jacques Schléret. Les téléfilms ont été écartés de cette étude. 


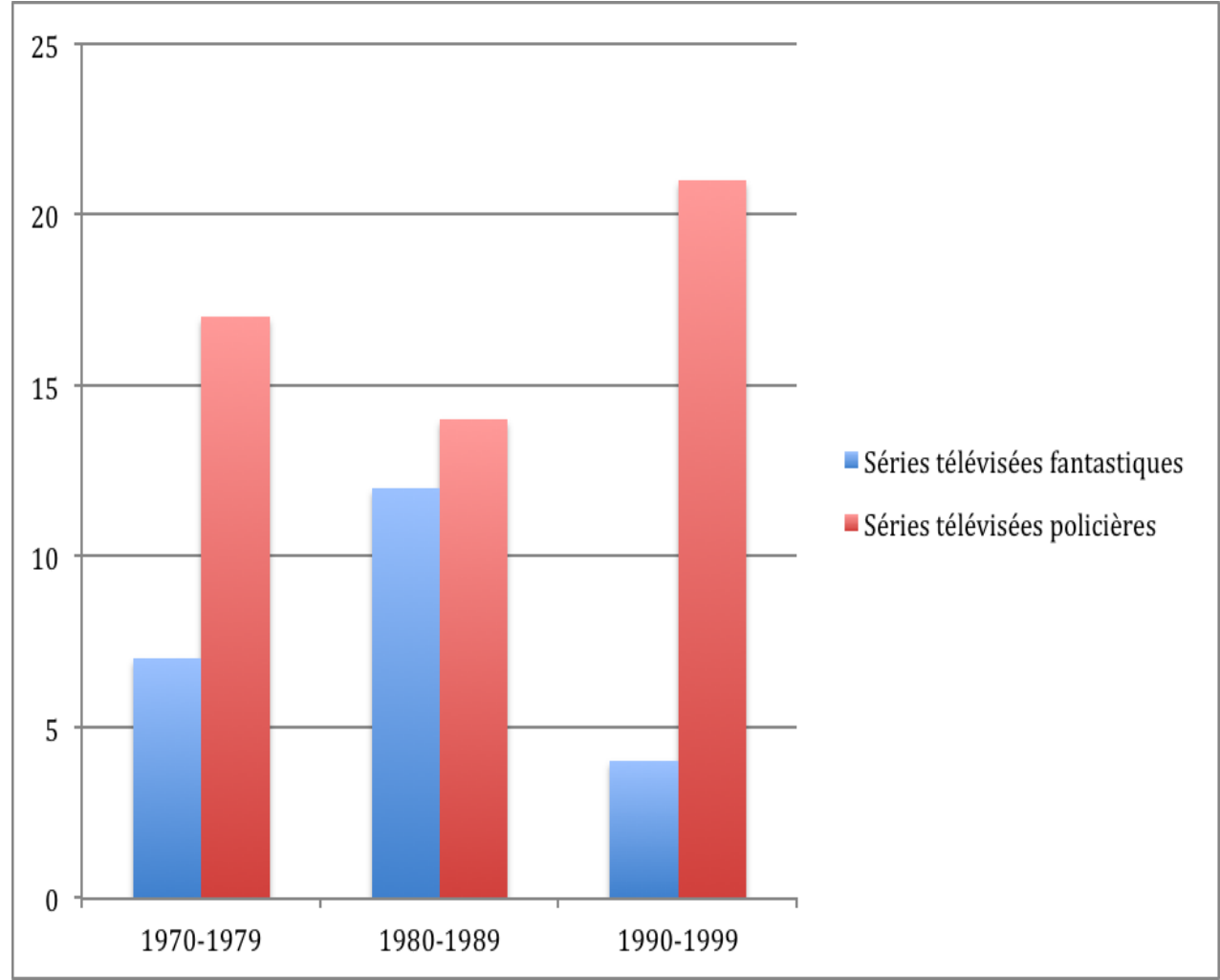

Fig. 2 : comparaison, par décennie, de la production des séries télévisées fantastiques et policières en France.

Notre propos ici se centrera autour de trois programmes diffusés sur un intervalle de cinq mois en 1971, ce qui correspond au début de la phase d'expansion des séries et feuilletons fantastiques sur les écrans télévisés français. Deux sont des séries hybridant le genre du policier et celui, soit de la franche science-fiction (Aux frontières du possible ${ }^{5}$ ), soit du fantastico-merveilleux ( $\mathrm{La}$ Brigade des maléfices ${ }^{6}$ ). Le troisième est un

${ }^{5}$ Aux frontières du possible est diffusé le lundi soir, en octobre et novembre 1971 sur la 2e chaîne de l'ORTF. Créée par Henri Viard et Jacques Bergier, elle narre les enquêtes d'un duo d'enquêteurs, Barbara Andersen et Yann Thomas, deux agents du BIPS, le Bureau International de Prévention Scientifique. Cet organisme a pour but d'empêcher que les découvertes scientifiques ne tombent entre de mauvaises mains (escrocs, terroristes, etc.). Indéniable succès public (une seconde saison fut commandée, qui fut diffusée en 1974), elle fut l'objet de peu de retours critiques dans la presse. L'INA édita, dans le cadre de sa collection "Inédits fantastiques », la première saison (6 épisodes) en 2012 et la seconde saison (7 épisodes) en 2013.

${ }^{6}$ La Brigade des maléfices a été créée par Claude Guillemot et Claude-Jean Philippe (critique de cinéma, animateur du ciné-club du dimanche matin au cinéma L'Arlequin). Elle compte six épisodes, diffusés le lundi soir du 2 août au 6 septembre 1971 sur la $2^{\text {e }}$ chaîne de l'ORTF. Chaque épisode montre d'abord l'échec des méthodes traditionnelles, l'appel à la Brigade des maléfices, puis l'enquête extraordinaire que mènent Guillaume-Martin Paumier et son assistant, Albert, face au scepticisme de Muselier. La série connut une 
feuilleton (selon la typologie de l'époque: aujourd'hui, on parlerait davantage de minisérie) de science-fiction et d'anticipation (Le Voyageur des siècles ${ }^{\top}$.

Lorsque l'on visionne ces programmes comme un téléspectateur des années 2000 rempli de préjugés sur la télévision française et éventuellement né après leur diffusion même, on ne peut être qu'étonné par l'inventivité et la fantaisie de ces épisodes. Et surtout, nourris que nous sommes des théories d'analyse de la télévision, ces programmes ne semblent pas tout à fait rentrer dans les cases que nous connaissons, telles que celles de paléo- et de néo-télévision ${ }^{8}$. En effet, leur inscription dans une forme de quotidien (en ce qui concerne La Brigade des maléfices et Aux frontières du possible) et leur réflexivité (surtout dans La Voyageur des siècles et La Brigade des maléfices), caractères de la néo-télévision selon les créateurs de ce concept, sont bien présents dans ces séries et feuilletons censés appartenir à la paléo-télévision. Faut-il faire l'hypothèse que cela est dû aux genres du fantastique et de la science-fiction, genres moins normés et plus ouverts que d'autres ? Peut-être, mais cela ne pourrait être vérifié que par des comparaisons fines avec d'autres genres, ce qui n'est pas notre propos ici. Il est par ailleurs évident que la rupture, si rupture il y a bien eu, ne s'est pas faite d'un coup et qu'il y a un continuum de pratiques dont les programmes qui nous occupent pourraient être des précurseurs. En outre,

exposition assez limitée, en partie à cause de sa diffusion estivale, mais fut remarquée par la presse (notamment par les deux grands critiques télévisuels du Monde de cette époque, Jacques Siclier et Martin Even). Elle fut redécouverte par les milieux sériphiles français en mars 2006 à l'occasion de la rétrospective INA accueillie par les 19e Rencontres Internationales de Télévision de Reims où l'épisode " La 7e Chaîne » fut projeté. L'INA sortit les six épisodes en DVD dans sa collection des Inédits fantastiques en 2011.

${ }^{7}$ Le Voyageur des siècles fut diffusé en août 1971 (le samedi soir) sur la $1^{\mathrm{e}}$ chaîne (en N\&B à l'époque, alors que la seconde chaine bénéficiait de la couleur). Les avis critiques sont très partagés (notamment parce qu'on regrette l'absence de critique sociale de l'anticipation et qu'on juge la réalisation et le parti-pris vieillots). L'histoire (en quatre épisodes) est complexe : en 1980, le comte Philippe d'Audigné, un scientifique doux-dingue, disparait brutalement. En réalité, il a réussi à opérer un voyage dans le temps, en ayant repris les théories de son arrière-grand-oncle, le comte François d'Audigné, qui travaillait à la fois sur la structure du temps et sur la réflexion des images du passé emprisonnées dans le tain des miroirs. Philippe rend visite à son aïeul, en 1885, ce qui lui permet d'apprendre l'identité d'une des femmes aperçues dans les reflets d'un miroir familial : il s'agit de Marie Dawson, la dame de compagnie de la comtesse Catherine d'Audigné, qui a disparu après que ses maitres ont fini sur la guillotine. Philippe en est tombé amoureux : Philippe et François partent alors en 1788 pour tenter de retrouver la suivante, mais leur voyage les amènera à changer le cours de l'histoire, en annulant la Révolution française grâce à d'avisés conseils transmis à Louis XVI. La série a été également l'objet d'une édition dans la collection des « Inédits fantastiques » de l'INA en 2012.

${ }^{8}$ Notions créées par Umberto Eco dans un article de presse, «TV: la transparence perdue » (republié dans Eco, 1985), elles ont été reprises par Francesco Casetti et Roger Odin (1990). Leur systématisation a cependant été vivement critiquée : voir par exemple Jérôme Bourdon (1998) ou Virginie Spies (2004). 
les travaux de Virginie Spies $(2004$; 2008) ont montré que la réflexivité est en réalité très ancienne à la télévision dans le domaine de l'authentifiant", massivement, et plus légèrement dans celui du ludique. Ces trois programmes montrent que la fiction n'était pas en reste et l'on retrouve des convergences entre ces trois mondes. Virginie Spies identifie quatre périodes dans l'histoire des émissions télévisées réflexives (c'est-à-dire des émissions prenant explicitement la télévision comme objet), dont les deux premières nous intéressent plus particulièrement :

- 1950-1970 : une télévision en quête de reconnaissance. Il s'agit d'une télévision qui recherche une forme de légitimité et une reconnaissance de la part du public en train de se former. C'est l'institution qui s'exprime à travers différents thèmes.

- 1970-1980: une interrogation de l'énonciation. Jean Frapat montre que les émissions réflexives peuvent prendre la forme d'une réflexion sur la télévision en tant que médium du point de vue de l'énonciation. Il ouvre de nouvelles perspectives à la réflexivité télévisuelle. (Spies 2008 : 9)

Il semble en effet que l'on puisse relire la réflexivité présente dans les fictions que nous étudions selon cette typologie : Le Voyageur des siècles, en effet, est assez proche d'un discours de reconnaissance de la télévision (notamment à travers tout un travail de filiation culturelle) tandis que $L a$ Brigade des maléfices joue clairement sur l'énonciation et les différentes possibilités de dialogue avec le public qu'offrent le médium télévisuel et la forme sérielle. Aux frontières du possible, pour sa part, est plus timide sur ces thèmes (la connivence passe davantage par un jeu avec l'actualité scientifique), mais quelques séquences mettent très clairement en avant un double discours sur l'énonciation.

\section{Le Voyageur des siècles : l'origine et le futur de la télévision}

Dans Le Vyyageur des siècles, la réflexivité passe par des moyens liés à l'anticipation et au voyage dans le passé. Le prologue du premier épisode, retranscrit ci-après et où aucune date n'apparaît (il faudra attendre plus de cinq minutes pour que le premier repère temporel, mai 1980, soit donné au téléspectateur), présente une nouveauté technologique : la télévision sans cadre.

"Une femme est assise dans un large fautenil design, dans un intérieur bourgeois. Elle tient une télécommande à la main et fume une cigarette. On entend le bruit d'un combat à l'épée hors champ. Contrechamp: un film de cape et d'épée est projeté sur le mur, on aperçoit en filigrane les murs du salon et un tableau de Louis $X V$.

Mme d'Audigné - Jolis réglages ! (Elle soupire)

\footnotetext{
${ }^{9}$ Sur la distinction authentifiant/ludique/fictif, voir Jost, p.28-31.
} 
Une sonnerie retentit. Mme d'Audigné se lève et va jusqu'à une console composée de plusieurs écrans et d'ampoules. Elle répond à l'appel en enclenchant un bouton. La tête d'un domestique apparaît sur un des écrans. Pendant le dialogue, on entend en hors champ les bruits du combat à l'épée.

Adrien - Conciergerie, Madame (Il enlève sa casquette). C’est Adrien. Madame, le Monsieur qu'attend Madame est là.

Mme d'Audigné - L'agence Boirot?

Adrien (avec une mimique d'ignorance et se tournant vers quelqu'un qui est hors champ) - L'agence Boirot?

M. Jolivet (entrant dans le champ) - Oui, Madame la baronne. Bonjour, Madame, je suis M. Jolivet.

Mme d'Audigné - Très bien, Monsieur, Adrien va vous mener jusqu'à moi. Ah, dites-moi, Adrien...

Adrien - Madame?

Mme d'Audigné - L’ouvrier de la télévision est passé ?

Adrien - Oui, Madame, il est venu hier.

Mme d'Audigné - Il n'a rien réparé du tout. C'est toujours aussi mauvais. Non. Vous auriez pu surveiller son travail. D'abord, l'appareil projette les personnages beaucoup trop gros, je n'arrive même pas à les réduire! Ils ont la tête dans mon plafond, c'est ridicule. On dirait des éléphants. Faites revenir votre bonhomme.

Adrien - Il dit que ... vous enclenchez pas... l'enclenchement sur... la bonne enclenchure... Enfin, pt'être!

Mme d'Audigné - Mais non... Pff... Mes enclenchements sont bons! Qu'est-ce qu'ils ont mes enclenchements? Et puis, voyons, la définition de l'image est trop faible! Tenez, en ce moment, sur la cinquième chaîne ${ }^{10}$, il y a une bataille dans une histoire du Moyen Âge quelconque. L'image se mélange avec les tableaux du fond de la pièce ! C'est immonde! En ce moment, le roi Louis XV est assis dans des mâchicoulis. C'est grotesque!

Adrien - Je ne comprends pas, Madame la baronne.

Mme d'Audigné - Bon, bon, ca ne fait rien. Introduisez M. Jolivet !

Adrien - Tout de suite, Madame. »

Le discours ici est intéressant à deux niveaux :

- la mise en scène de la télévision du futur ${ }^{11}$ telle que l'imaginent Noël-Noël et Jean Dréville dans cette fiction :

\footnotetext{
${ }^{10}$ Rappelons qu'en 1971, le paysage audiovisuel français ne compte que deux chaînes.

11 Virginie Spies (2008 : 5-6) indique que ce thème avait déjà fait l'objet d'une programme réflexif le 13 mars 1947 : «Intitulé Télévision : œil de demain, il présente d'abord la télévision de cette époque avant de s'envoler rapidement vers la fiction avec plusieurs saynètes qui imaginent d'une manière ludique ce que pourra être cette télévision de demain. Il s'agit en fait d'imaginer ce média du futur en expliquant que chacun disposera d'un récepteur de poche, et que télévision et téléphone ne feront plus qu'un. Télévision : œil de demain met en scène une fiction d'ordre ludique et l'énonciateur, une institution en devenir, possède déjà une conscience de ce que la télévision pourrait être dans l'avenir. » Il nous semble que nous retrouvons quelque chose de cet ordre, dans cet extrait, d'autant plus que le projet de
} 
«sans cadre ${ }^{12} »$, c'est-à-dire sans écran (bien que la télécommande ait toujours un fil), et étonnamment complexe à régler ;

la métaphore que la télévision sans cadre offre de la fiction que nous allons voir: Louis XV incrusté dans les mâchicoulis, soit le temps totalement mélangé, est annonciateur $\mathrm{du}$ bouleversement $\mathrm{du}$ temps que va montrer le feuilleton, avec par exemple le corps de Philippe d'Audigné trouvé totalement décomposé en 1981 et dont les spécialistes datent la mort à la fin du $18^{\mathrm{e}}$ ou au début du $19^{\mathrm{e}}$ siècle, alors qu'on l'a vu vivant en mai 1980.

Le feuilleton joue également avec la croyance du téléspectateur, avec le problème de la suspension consentie de l'incrédulité, explicitant à plusieurs reprises la question du vraisemblable ${ }^{13}$ et la référence à Jules Verne $^{14}$. Le texte du programme s'auto-commente à travers les dialogues des personnages, jouant d'une forme de métalepse. Ainsi, lorsque Philippe d'Audigné se présente à son aïeul, il utilise le vocabulaire de l'écriture fictionnelle : «Le dialogue qui va s'engager entre nous, mon cher maitre, embarrasserait bien un romancier qui aurait à l'écrire, parce qu'il aurait du mal à rendre la conversation vraisemblable, et qu'il passerait tout de suite auprès de son lecteur pour un dément ou pour un idiot. " Le discours d'autocommentaire passe aussi par des références à ce qu'on peut considérer comme deux ancêtres de la forme sérielle ou feuilletonesque télévisuelle : le roman-feuilleton et le cinéma. Les références au romanfeuilleton passent d'abord par le dispositif narratif utilisé : deux époques servent de récit-cadre (1981, année de la disparition de Philippe d'Audigné, et 1885 , moment où disparaît François d'Audigné). Le récit des aventures

Noël-Noël, datant des années 1930 et mettant plusieurs décennies à se concrétiser, relève du même type d'imaginaire.

${ }^{12}$ Cette notion de « sans cadre » peut aussi se comprendre « sans limites » et, dans un sens second, relever d'une forme d'autocommentaire: cette œuvre télévisuelle est sans cadre, totalement libre dans ce qu'elle propose. Rappelons que, pour Barthes (1976), la sciencefiction, plus que «l'invention de gadgets cosmiques ou physiologiques", est «la substitution perverse de l'imaginaire du désir à l'imaginaire de la vérité. »

13 Parmi un nombre important d'occurrences de mots liés au champ lexical de la vraisemblance et de l'aventure, nous pouvons relever les quelques exemples suivants : «Nous sommes plongés dans l'extraordinaire, n'est-ce pas ? Et bien, vautrons-nous dans l'extraordinaire!»; «Voyez, j'explique au roi toute notre aventure.»; «Dans notre randonnée, maittre, tout est invraisemblable, mais vrai. Il faut s'y faire ». Nous retrouvons ici une dichotomie bien connue, depuis Boileau ou la querelle du Cid, et même Aristote!

${ }^{14}$ Le feuilleton est lui-même qualifié dans le générique de "Julvernerie moderne ». De même, l'un des savants, amis de François d'Audigné, commente ainsi le récit lu par le secrétaire : "Je suis encore un peu circonspect. Enfin... La mode est à Jules Verne. Allons-y!» 
de Philippe et de François est une lecture faite par le secrétaire de François à un groupe de savants amis de ce dernier, lecture qui sert d'embrayage aux analepses allant de la veille de la Révolution française jusqu'à l'époque napoléonienne. Les épisodes sont donc rythmés par ces scènes de lecture, réparties sur plusieurs journées, qui peuvent offrir des passages réflexifs, comme ce dialogue qui clôt à la fois une journée de lecture et un épisode : «Oh, mais Messieurs, Messieurs, n’anticipez pas! Attendez la suite! - La suite... - La suite au prochain numéro !», reprenant la formule canonique de ce type de narration et inscrivant le feuilleton télévisé dans les traces du roman-feuilleton. La référence est parfois encore plus directe, comme dans ce dialogue précédant une séance de lecture : «Eh bien, si nous allions écouter la suite. - Ah ah! Notre roman-feuilleton, comme vous dites, Maitre, vous intéresse. - J'ai toujours été un peu midinette. » Les références au cinéma, quant à elles, sont moins structurantes et plus éparses, partagées soit de manière directe ${ }^{15}$, soit de manière indirecte par des mentions de formes de spectacles lui ayant préexisté ${ }^{16}$.

Tous ces phénomènes livrent une explicitation du geste de l'écriture qui, d'une certaine façon, rompt la transparence et insiste sur le caractère non seulement fictionnel, mais encore proprement télévisuel de ce que nous regardons. Mais ces clins d'œil ne sont pas les seuls éléments qui jouent de la relation entre le programme et le public. L'invention d'un futur proche, mais très décalé, le voyage dans le passé de deux personnages issus de deux temporalités différentes, et ayant donc deux cultures différentes, la réécriture uchronique du début du $19^{\mathrm{e}}$ siècle, tout cela va instaurer un dialogue entre ce que le public sait et ce que la fiction propose. À plusieurs reprises, notamment dans les moments narrés à la $1^{\mathrm{e}}$ personne par l'aïeul ${ }^{17}$, le téléspectateur est celui qui saisit la totalité du sens, face à François d'Audigné qui ne peut pas comprendre parce qu'il ne maitrise pas les références culturelles ou historiques maniées par son arrière-grand-neveu. Par exemple, lorsque les deux héros rencontrent La Fayette et que ce dernier leur indique vouloir, l'année suivante, lancer des fouilles archéologiques sur ses terres et faire creuser des galeries, Philippe

\footnotetext{
${ }^{15}$ Un des personnages, incrédule, déclare à l'enquêteur qui tente de reconstituer l'épopée de Philippe d'Audigné, "Vous me faites du cinéma-fiction, tous les deux !», exclamation renforcée par un gros plan sur le visage de ce personnage

${ }^{16}$ On relève la lanterne magique, de même que le chanteur de complaintes (que l'on voit psalmodier tout en pointant d'une baguette des gravures représentant les différentes étapes de son récit), pratique que Philippe d'Audigné commente ainsi «Voilà le chanteur de complaintes, apaisant encore ici chez nos pères leur soif inconsciente de cinéma ». Il faut noter la vision téléologique ici exprimée et une forme de positivisme assez surprenante dans une œuvre de 1971, qui peut s'expliquer par la conception ancienne du projet.

${ }^{17}$ Rappelons que la manière dont le récit de François d'Audigné est livré au téléspectateur est une lecture a posteriori donnée par son secrétaire.
} 
d'Audigné commente «Alors j'ai pensé qu'au Printemps prochain, on aurait les Galeries La Fayette! [...] C'est une plaisanterie Bon Marché ». Lors de leur voyage dans le temps, ils rencontrent également un de leurs ancêtres qui leur demande leur identité lorsqu'ils les trouvent sur son domaine :

«Philippe - Nous-mêmes sommes affiliés par notre arrièregrand-mère à la branche irlandaise. Je vous présente donc ainsi mon vénéré oncle, le comte Bob Dylan.

Secrétaire de François (en voix off) - Il devait y avoir là une de ces malices dont Philippe avait le secret.

Philippe - Je me présente moi-même, comte Winston Churchill.

Secrétaire de François - Ces pseudonymes qu'il nous avait choisis semblaient l'amuser beaucoup. Sacré Philipe. »

Les décalages temporels entre les contemporains de François d'Audigné et le public vont aussi permettre la création d'une connivence, même si elle nécessite parfois une bonne dose de culture historique! Ainsi, Philippe est parti dans son voyage dans le passé avec une Histoire de France illustrée qu'il appelle son «guide Michelet» (contraction du guide Michelin et de Jules Michelet). Le secrétaire de François d'Audigné n'arrive pas toujours à déchiffrer l'écriture de son maître et, en voyant dans la même phrase le nom du général Boulanger et le mot Bonnemains, tous font l'hypothèse que, avec le général Boulanger, la France est entre de bonnes mains (alors que c'est le nom de la maittresse du général, sur la tombe de laquelle il se suicide). Ce même type de jeu existe dans le décalage entre le public et le futur inventé pour 1981: Georges Brassens entre à l'Académie française et les gendarmes lui font une haie d'honneur; Jean Marais est doyen de la Comédie-Française (institution qu'il a quittée à deux reprises, en 1941 et en 1952), par exemple.

Un autre clin d'œil est fondé sur la présence de La Fayette. Jean Dréville, dix ans avant Le Voyageur des siècles, avait tourné un film consacré à la vie de cette figure historique. Lorsqu'il retrouve ce personnage dans le feuilleton, il rappelle le même acteur, Michel Le Royer, qui incarne de ce fait deux fois le même rôle, dans deux œuvres du même réalisateur, à dix ans d'intervalle: nous retrouvons ici ce que les Américains appellent le cameo.

\section{La Brigade des maléfices et Aux frontières du possible: s'amuser en écrivant}

Le programme qui met le plus au cœur de son écriture la réflexivité est La Brigade des maléfices, qui renvoie à plusieurs reprises, et de manière malicieuse, à ses propres conditions d'énonciation. Le cas le plus flagrant est celui du deuxième épisode de la série, « $\mathrm{La} 7^{\mathrm{e}}$ Chaîne », qui place au centre de l'enquête un revendeur/loueur de téléviseurs et le Diable luimême, qui occupe la posture d'un producteur de programmes tout à fait 
particulier ${ }^{18}$. En effet, cet épisode narre les mésaventures d'un jeune couple qui installe chez lui un téléviseur prétendument expérimental proposant les programmes de la septième chaîne. Rappelons qu'à l'époque, seules deux chaînes existaient (la troisième sera lancée l'année suivante), la série se place par conséquent totalement dans un univers de fantaisie. Ce téléviseur est aussi particulier en ce qu'il comporte une caméra embarquée qui permet à Diablevert de voir ce qui se passe chez le jeune couple. Cela lui permet d'adapter la situation du feuilleton qu'il produit à ce qui se passe en réalité devant le téléviseur, dans le but de manipuler son public et de pousser l'homme, par jalousie, à tuer son épouse. Le discours tenu sur la télévision est intéressant en ce qu'il semble assumer, tout en le repoussant ironiquement, l'idée d'une influence de la fiction sur le public, surtout quand elle se donne pour but de divertir. N'oublions pas qu'à cette période, les discours de ce type ne sont pas rares; cette décennie verra par exemple la publication de La Propagande inavouée de Jean-Marie Piemme ${ }^{19}$.

En outre, le feuilleton produit par Diablevert semble faire référence, par la mise en scène et l'opulence des personnages, aux soaps américains ${ }^{20}$ qui ne sont cependant, à notre connaissance, pas diffusés à cette époque sur l'ORTF ${ }^{21}$. Le discours est de ce fait à la fois distant (par la référence

\footnotetext{
18 Virginie Spies $(2008,6)$ indique que l'ORTF programmait, de 1965 à 1972, une émission se penchant «sur les métiers de l'audiovisuel, et plus particulièrement ceux de la télévision », Au-delà de l'écran. : "L'ambition principale de cette émission réflexive est de faire découvrir, sur un ton très sérieux, la télévision aux téléspectateurs. »

${ }^{19}$ Le projet du livre est clairement énoncé dans sa quatrième de couverture, qui reprend une rhétorique marxienne assez spécifique de l'époque: "Comme la littérature commerciale, le feuilleton télévisé ne constitue pas un univers fermé sur lui-même qui n'aurait rien à voir avec la vie de tous les jours, une espèce d'enclos sans aucune réalité politique. C'est au contraire un élément engendré dans et par une société donnée. Plus précisément, c'est un lieu de cristallisation idéologique où se réfléchit une époque et où la "multitude silencieuse" apprend à parler un langage qui aide à sa domination par la classe dominante. »

${ }^{20}$ Il faut noter que Jacques Baillon, un des créateurs de la série, a revendiqué très explicitement une influence américaine sur sa façon de travailler. Dans un article de 1976), qui dit que La Brigade des maléfices est « un feuilleton à la française qui a [...] les qualités d'un feuilleton à l'américaine », Jacques Baillon déclare " J'ai bien étudié les séries. Je les ai même magnétoscopées. Au fond, c'est simple : la série, c'est des gros plans, des gueules, un bon scénario. De la nervosité dans le montage et de l'efficacité. Nous nous obstinons à tourner des histoires banales sur de grands plateaux avec de grands décors. Tout ça pour un petit écran! » (Cet article sans indication ni de date ni d'origine, intitulé «La Brigade des maléfices tous les jours sur TF1, 14h15. Un bon sort...» et signé Michèle Stouvenot, est disponible dans le dossier 45 du carton 15007-26 du Fonds TF1 de l'INA; le contenu de l'article permet d'inférer qu'il a été publié lors de la deuxième rediffusion de la série sur TF1 en 1976.)

${ }^{21}$ Cela ne veut pas forcément dire qu'ils étaient totalement inconnus en France : même s'ils ne couvraient pas tout le territoire, Télé-Luxembourg et Télé Monte-Carlo ont pu en diffuser et surtout, des magazines comme Radio-Cinéma, devenu par la suite Télérama en
} 
culturelle) et proche (par le rapport que la série montre entre le programme mis en abyme et son public, le couple de jeunes mariés) : une forme de familiarité, pour le public réel de 1971, est instaurée. Si nous analysons la scène de la première soirée que le couple passe à regarder les programmes expérimentaux de la septième chaîne, nous voyons comment tout est fait pour que les deux espaces se répondent: alors que le jeune couple dîne devant le téléviseur, les deux personnages du feuilleton passent aussi à table et Diablevert, qui observe le jeune couple, fait rejouer geste par geste la scène quotidienne par son duo d'acteurs incarnant un couple grand bourgeois. La scène est filmée de telle façon que le téléviseur, à l'arrière plan, crée une répétition de la scène au premier plan (voir figures 3 et 4).

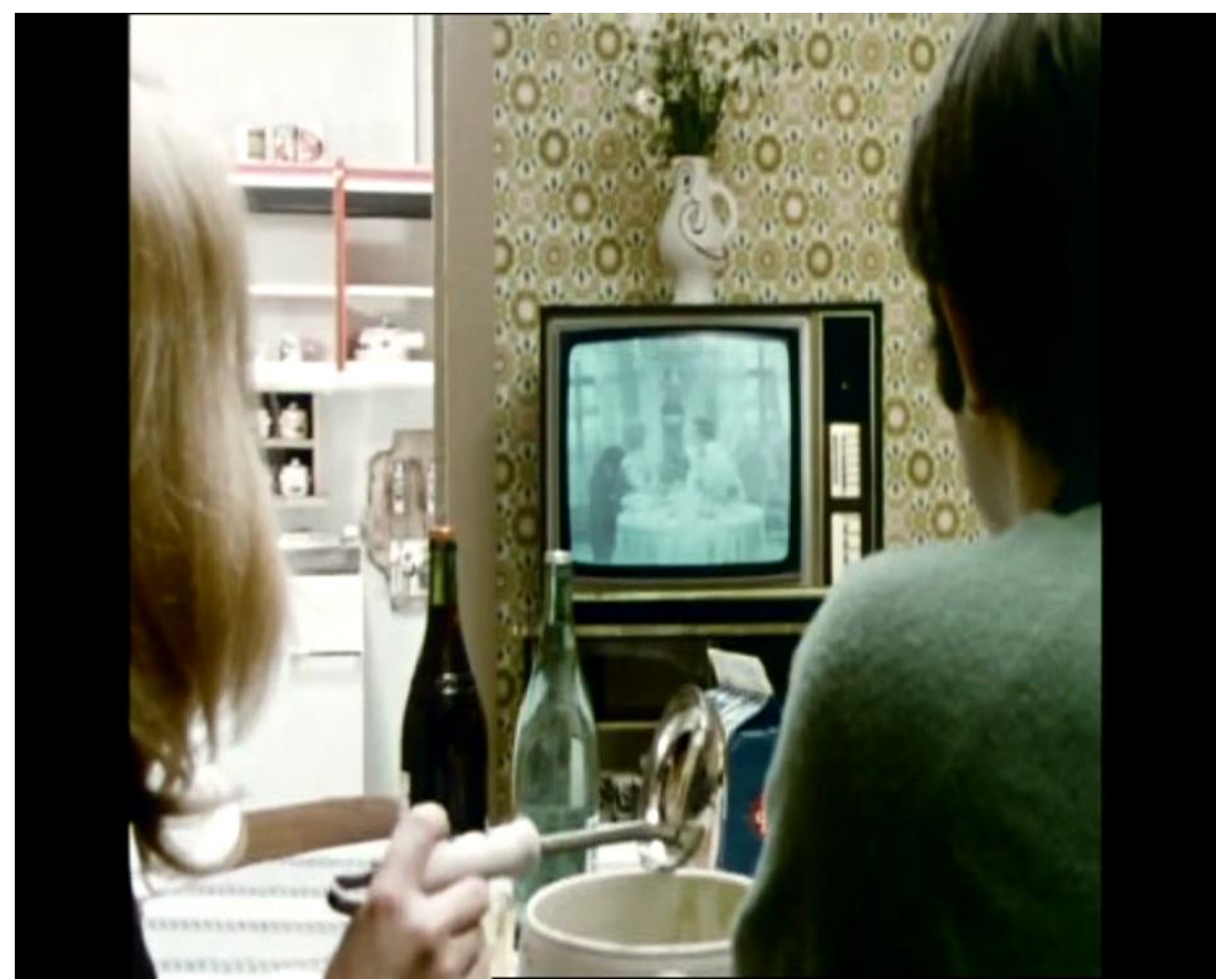

Fig. 3 : La Brigade des maléfices, «La $7^{\circ}$ Chaîne ». (DR)

Mais le travail d'entrelacement est encore plus prononcé sur le plan sonore. Une réplique du feuilleton du téléviseur trouve ainsi sa réponse dans le dialogue entre les deux jeunes mariés : ma chère?

«Acteur - Délicieux, ce velouté de tomates, vous ne trouvez pas

Jeune mariée - Oh, tu sais, c'est de la soupe en sachet.

Jeune marié - Ça ne fait rien, je trouve ça très bon. »

1960, proposaient parfois des reportages sur la télévision à l'étranger et pouvaient en conséquence aborder des programmes ou des genres inédits chez nous. 


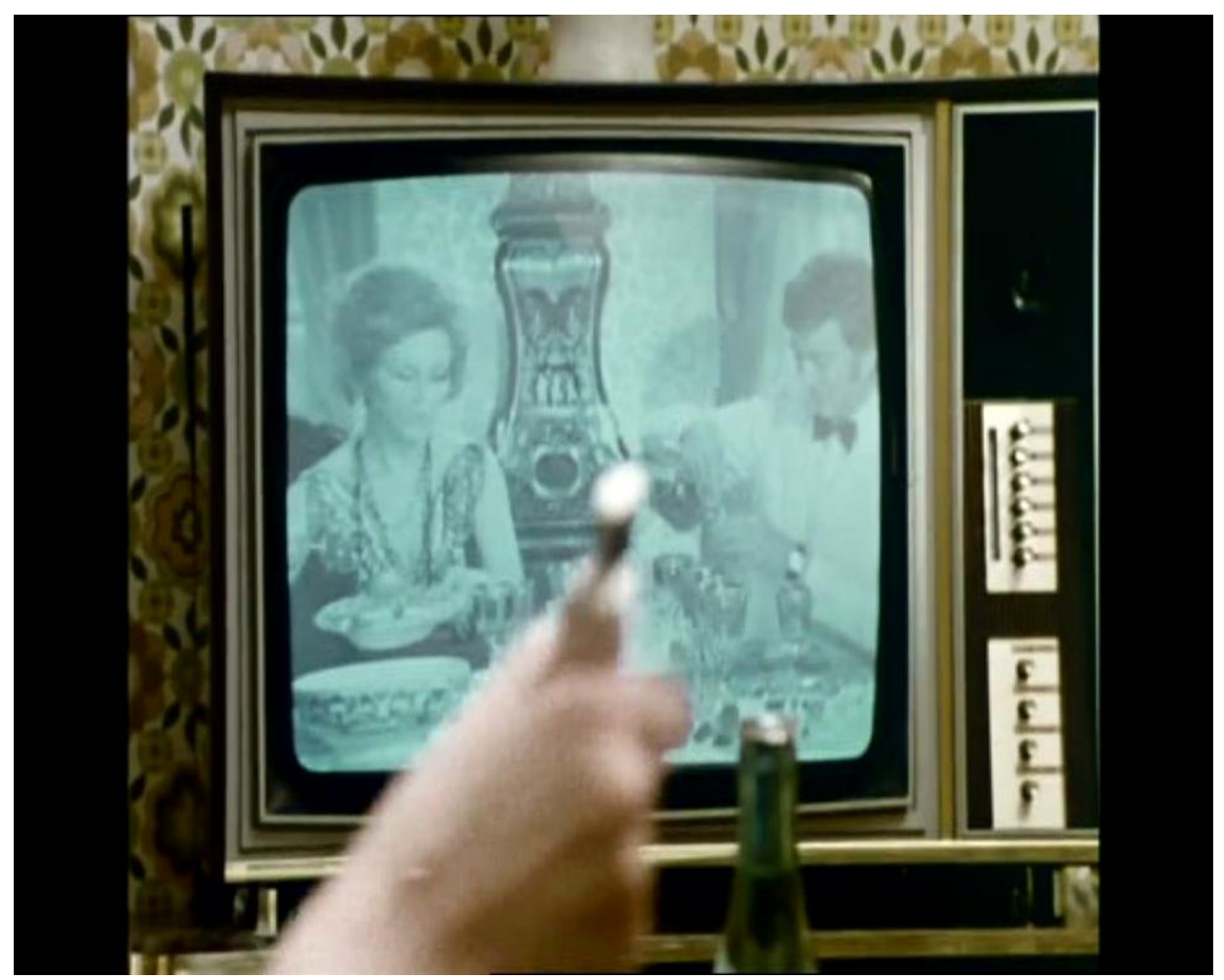

Fig. 4 : la soupe est servie en même temps dans les deux espaces diégétiques. (DR)

Ce procédé était déjà présent dans la séquence d'introduction de l'épisode, celle qui évoque le meurtre qui va provoquer la saisine de la Brigade des maléfices pour résoudre l'affaire. L'épisode commence par un mouvement tout cinématographique: la caméra se trouve devant une façade d'immeuble, avec une succession de fenêtres qui figurent comme des écrans dans l'écran, elle monte et s'approche d'une fenêtre aux stores baissés ${ }^{22}$, s'arrêtant avant de pénétrer dans l'appartement. On entend alors une détonation et, sans transition, le téléspectateur est placé devant un couple regardant un western à la télévision. Le dialogue du film s'interroge sur la réalité ou non d'un coup de feu : le téléspectateur est alors amené à se demander si cette détonation appartient à la bande-son de la série qu'il regarde (adéquation entre la bande-image et la bande-son) ou à celle du western regardé par les personnages (anticipation de la bande-son de la seconde séquence sur les images de la première séquence).

\section{«Bruit de détonation}

Personnage de western $1-\mathrm{Oh}$, Bill, t'as entendu?

Personnage de western 2 - Quoi, Joe?

\footnotetext{
${ }^{22}$ C'est le genre de mouvements de caméra que l'on trouve, par exemple, en ouverture de Psycho d'Hitchcock.
} 
Personnage de western 1 - Un coup de feu (Une musique typique de western commence et se poursuivra tout au long de la séquence)

Personnage de western 2 - Écoute, vieux Joe, tu vois bien qu'il n'y a personne. Le désert est vide, comme d'habitude. Allez, faut dormir... Nous avons un sacré bout de chemin à faire demain. Tu l'as rêvé ce coup de feu. coup de feu.

Mari de la concierge - Oh, c'est bizarre, je l'ai entendu aussi ce

Concierge - C'était pas un coup de feu, c'était une porte qui claque au 3ème.

Mari de la concierge - Non, mais enfin, je t'assure. J'ai entendu une détonation. (La femme hausse les épaules). Mais enfin, puisque je te le dis... (On cogne à la porte). Y a pas de raisons que...

Concierge - Qu'est-ce que c'est encore? Ils ne peuvent pas nous laisser tranquilles. C'est plus fort qu'eux. (Elle entrowrre le rideau de sa porte pour voir qui se trouve derrière, puis l'ouvre). M. Rochemont? Mais qu'est-ce que vous avez, vous êtes tout pâle?

Mari de la concierge - Qu'est-ce que c'est ?

M. Rochemont - J'viens de tuer ma femme. (Le mari recule, sa femme le rejoint)

Concierge (en chuchotant) - C'est M. Rochemont, du 3ème, il vient de tuer sa femme.

M. Rochemont (hors champ) - Voudriez-vous s'il vous plait téléphoner à la police ?

Concierge - Oh mais oui. Bien sûr. Tout de suite, M. Rochemont (elle sort, le mari reste seul, puis la suit).

M. Rochemont - Merci (pendant la suite du dialogue, M Rochemont, très lentement, s'installe devant le téléviseur et pose son revolver sur la table basse)

Mari de la concierge (hors champ) - J't'avais bien dit que j'avais entendu un coup de feu. J'suis pas fou, tout de même.

Concierge (hors champ) - Oh toi, tu veux toujours avoir raison!

faut. »

Mari de la concierge (hors champ) - Alors là, je ne sais pas c'qui te

La réalisation et l'écriture de cette séquence mettent en place, dès le début de l'épisode, une forme de dialogue entre l'espace diégétique et l'espace fictionnel ouvert dans l'image par le poste de télévision qui sera poursuivi, par divers dispositifs, tout au long de la narration. Ainsi, à la toute fin de l'enquête, Muselier, le commissaire sceptique de la PJ, manifeste son mécontentement face à la date choisie pour l'arrestation de Diablevert car c'est le jour de son feuilleton. Comme ils veulent prendre Diablevert en flagrant délit, tous les policiers seront mobilisés à l'heure de grande écoute, soit sur le terrain, soit dans le grenier qui sert de repaire à la Brigade des maléfices où Muselier et Paumier regardent la septième chaîne pour pouvoir déterminer le moment le plus adéquat pour donner l'assaut. Heureusement, une fois l'opération finie, Paumier change de chaîne pour que Muselier puisse voir son feuilleton. La musique du générique de fin de La Brigade des maléfices commence en anticipant sur la dernière séquence de l'épisode. Cette fois-ci, le discours de la fiction et celui de l'énonciation 
extradiégétique se mêlent. Nous sommes passés d'une porosité restreinte à l'intérieur de la fiction à une porosité entre le monde de la fiction et le nôtre.

«Paumier règle le téléviseur sur la chaîne du fenilleton que Muselier veut voir. Muselier - Ça doit être fini. Enfin, je ne vous en veux pas.

Paumier et Muselier s'installent devant le téléviseur. La musique du générique de La Brigade des maléfices commence.

Paumier - Ah... Bah... (Il fait un geste d'impuissance en direction du téléviseur) C'est la fin, oui.

Muselier - J'aurai toujours le générique. Ça va me donner des regrets.

Carton habituel du générique de fin: "image DENYS CLERVAL assisté de NOEL VERY». Second carton : "son JEAN LOUIS UGHETTO perchman DOMINIQUE DALMASSO ». Retour dans le grenier.

Muselier - Vous avez vu? Mais quelle équipe!

Carton: "décor JEAN LOUIS DHUIT ensemblier HENRI OFFROY». Second carton: "régisseur général PIERRE ANDRIEUX assistants réalisateurs JEAN YVES ROUSSEAU JEAN ROGER $S A H U N E T$ script-girl BEATRICE MORCH ». Retour dans le grenier.

Muselier - Vous savez, les feuilletons, c'est comme tout. Il y en a beaucoup de mauvais. Mais quand ils sont bons... télévision.

Paumier - En tout cas, c'est bien le meilleur qu'on ait vu à la

Le générique se poursuit comme à l'accoutumée.»

L'autocommentaire passe ici par un dialogue entre deux espaces normalement étanches (celui de la fiction et celui du monde réel) et sert de support à un discours idéologique sur la télévision, quasiment de justification, sur un mode ludique qui ne se prend pas au sérieux ${ }^{23}$. Dans le cas de cet épisode, le discours est massif : aucun téléspectateur ne peut passer à côté. Mais il y a dans la série d'autres exemples, plus ponctuels et qui ne sont repérables que par des téléspectateurs plus attentifs. Ainsi, dans le dernier épisode de la série, voici ce que l'on voit et entend au milieu de l'épisode.

\footnotetext{
23 À ce propos, il nous semble amusant de rapprocher ce dispositif de la conclusion d'un des articles que La Provence a consacré à la série au moment de sa sortie : «Je pense aussi qu'il convient d'encourager Claude Guillemot et Claude Nahon à poursuivre leur expérience qui consiste à écrire pour la télévision en fonction de ses possibilités. Ce n'est, en effet, pas si fréquent.» (Ferré 1971). Si l'article pointe plutôt l'absence de grandiloquence et une esthétique se démarquant de celle du cinéma, il nous semble que nous pouvons aussi lire cette phrase à rebours en soulignant la polysémie de l'expression «en fonction de ses possibilités» qui peut vouloir dire «en exploitant toutes ses possibilités».
} 


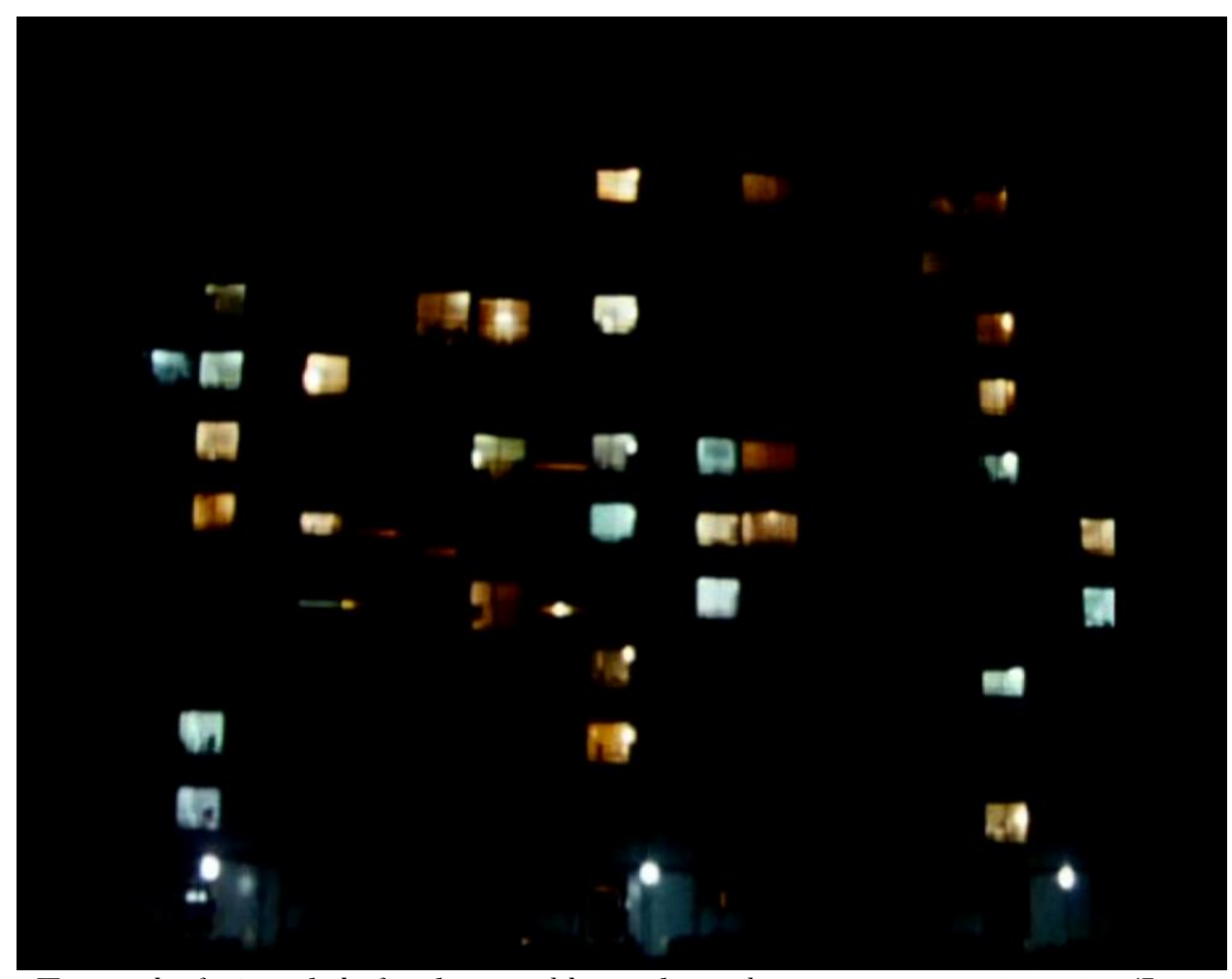

Fig. 5 : les fenêtres de la façade ressemblent à de nombreux écrans mis côte à côte. (La Brigade des maléfices, «Le Fantôme des HLM », DR)

«Une façade d'immeuble, de nuit. Certaines fenêtres sont allumées. On en voit certains s'éteindre. Le cadrage et l'exposition sont tels qu'on a l'impression d'être devant un mur de téléviseurs ${ }^{24}$. Une musique douce au clavecin accompagne les images.

Speakerine (en voix hors champ) - Mesdames, mesdemoiselles, messieurs, nos émissions sont maintenant terminées. (Cadrage sur l'écran $d u$ téléviseur) J'espère que vous avez passé une agréable soirée en notre compagnie. (Cadrage sur un homme en manches de chemise et gilet, affalé dans un canapé en cuir). Je vous rappelle que demain, sur la $2^{\mathrm{e}}$ chaîne, à $20 \mathrm{~h} 30$, vous verrez le 49e épisode de La Brigade des maléfices, "La Sorcière du drugstore ». (L’homme se lève). Maintenant, je vous souhaite une très bonne nuit. Bonsoir.

L'homme éteint le téléviseur, fait le tour de la table basse et va réveiller sa femme endormie à l'autre bout du canapé.

Homme - Eh! Ohoh!

Femme - Qu'est-ce qu'il y a ?

Homme - C'est fini. Allez (il s'éloigne). Au lit. (La femme range le tricot qu'elle avait sur les genoux, se lève et suit son mari). "

\footnotetext{
${ }^{24}$ Nous retrouvons ici un cadrage et une formalisation visuelle proches du début de « La

7 e Chaîne » de cette même série, dont nous avons parlé précédemment.
} 
L'ironie d'annoncer un $49^{\mathrm{e}}$ épisode (longévité exceptionnelle pour une série française, a fortiori à cette époque) alors que la série s'arrête ici, sur son sixième épisode, est savoureuse. De même, le titre annoncé, «La Sorcière du drugstore », est un décalque de celui de ce même épisode, «Le Fantôme des HLM» (nom désignant une figure folklorique $+d e+$ nom connotant la modernité). Enfin, le «C'est fini» est lui-même à double entente : les programmes sont finis dans la diégèse et la série, dans notre vie de téléspectateur de 1971, touche à sa fin. Tous ces éléments ne sont pas au même niveau, mais montrent cependant la distance des auteurs et leur plaisir à multiplier les clins d'œil à l'attention du téléspectateur.

Nous sommes face à un cas totalement différent avec Aux frontières du possible. La série joue moins clairement la carte de la réflexivité. En revanche, elle instaure un dialogue permanent entre les réalités scientifiques, les thèmes de recherche dans l'air du temps, les frémissements des applications militaires, en d'autres termes la réalité qui environne le téléspectateur, et les enquêtes menées par le BIPS (le Bureau International de Prévention Scientifique, l'agence qui emploie Barbara et Yann). Qu'il s'agisse de la synthèse des diamants (les premiers diamants synthétiques datent de la moitié des années 1950, et la fin des années 1960 voit de nombreux essais pour tenter d'améliorer le processus), de la conquête de l'espace (l'homme n'a marché sur la Lune que depuis deux ans), des améliorations dans le guidage des avions, les questions auxquelles sont confrontés Yann et Barbara ne sont fondées que sur l'idée du "un pas de plus par rapport au niveau de connaissance actuel». Ce faisant, elles permettent au public d'identifier des problématiques plus ou moins connues de lui, dont on parle dans les journaux, et d'ouvrir à partir de là un espace fictionnel démarqué de notre monde, mais qui y est en même temps solidement lié.

Une bonne illustration de ce principe est l'épisode «Protection spéciale ultra-sons U », dont l'intrigue est fondée sur les capacités des ultraultra-sons (dits aussi ultrasons $U$, ils seraient un type d'ultrasons extrêmement puissants). Ces derniers ont la capacité de "capter les humains en images et en sons ", autrement dit, d'une certaine façon, de les filmer. De là, une bonne partie du texte de l'épisode est interprétable en termes de métadiscours sur la télévision. Tout d'abord, les retombées technologiques des ultra-sons $U$ étant une amélioration de la diffusion télévisée, une partie de l'enquête va se dérouler dans ce domaine : Barbara se fait passer pour une journaliste écrivant un dossier sur «L'audiovisuel dans le monde de demain» pour entrer dans une usine de transistors spéciaux qui sont partie prenante de la fabrication de «télévisions minces comme une feuille de papier et grandes comme un mur ». L'enjeu principal de l'enquête est le secret gardé autour d'une conférence internationale que des malfaiteurs menacent de rendre publique grâce à une captation par ultra-sons U. Ces derniers contactent le BIPS pendant une réunion de 
travail et leur font comprendre qu'ils les voient, en ce moment même, à travers des allusions à leurs postures, leurs gestes, etc. $\grave{A}$ une remarque sur la robe de Barbara, cette dernière demande que les malfaiteurs lui disent de quelle couleur elle est: «Bonne question, Mademoiselle! Mais vous me faites beaucoup d'honneurs. Notre procédé n'est pas encore en couleurs. Il le sera bientôt, j'espère ! Or, en 1971, au moment de la diffusion de cet épisode, si la $2^{\mathrm{e}}$ chaîne (qui diffuse la série) est bien en couleurs, la $1^{\mathrm{e}}$ chaîne, elle, émet toujours en noir et blanc ${ }^{25}$. On note cependant que le fonctionnement du texte est ici bien différent de celui de « $\mathrm{La} 7^{\mathrm{e}}$ Chaîne » de La Brigade des maléfices: le texte est plus sibyllin, avec un contenu implicite destiné à des initiés capables de mobiliser un double registre de lecture.

Cette opacité possible de la référence est également présente dans l'épisode "Attention, nécroses mentales », où l'on entend une réplique $a$ priori anodine: "Non, mais cette théorie est absurde. Votre histoire de vitesse est du domaine des bandes dessinées pour adultes attardés! ». Elle peut certes être interprétée comme un commentaire réflexif amusé sur le statut de divertissement de la série (d'autant plus que cette hypothèse sera confirmée plus tard), mais elle ne prend son sens plein que si on la rapproche de Jacques Bergier, le créateur de la série: ingénieur chimiste diplômé de l'École Nationale Supérieure de Chimie de Paris, spécialiste du nucléaire $^{26}$, ancien Résistant, prétendument ancien agent secret, écrivain ${ }^{27}$, l'homme ne manque pas de casquettes! Disciple de Charles Fort - un scientifique américain du début du $20^{\mathrm{e}}$ siècle qui s'est attaché à la description et à la compréhension des phénomènes inexpliqués tels que les pluies de grenouilles, les observations d'OVNI, les vestiges archéologiques lilliputiens, etc. - Jacques Bergier, avec l'aide de Pauwels, crée ce qu'on a appelé le réalisme fantastique, fondé sur l'idée que la science se ferme aux phénomènes qui bouleversent ses conceptions et qu'il faut en conséquence aller vers ces phénomènes pour tenter de les comprendre en eux-mêmes et pour eux-mêmes. D'une certaine façon, c'est ce que font les enquêteurs du BIPS, qui se trouvent plus d'une fois face à des phénomènes inexpliqués qu'une avancée technologique inconnue du grand public ou une nouvelle découverte scientifique rend plausibles. Mais comment lier cette mention de «bandes dessinées pour adultes attardés » et la personnalité de Jacques Bergier? C'est que Jacques Bergier a lui-même inspiré des personnages de

${ }^{25}$ Elle ne commencera à émettre certains de ses programmes en couleurs en utilisant le canal de FR3 qu'en 1975, et il faudra encore attendre un an pour que des émetteurs permettant la diffusion en couleurs commencent à être déployés, en région parisienne, pour TF1. Les émetteurs noir et blanc ne cesseront leur activité qu'en 1983.

${ }^{26}$ Il réussit la première synthèse naturelle d'un élément radioactif et est l'un des découvreurs du rôle de l'eau lourde dans le freinage des électrons.

${ }^{27}$ Auteur, avec Pauwels, du Matin des Magiciens, il est aussi un ami de Jean Bruce (le créateur d'OSS 117) et de Ian Fleming. 
scientifiques dans des bandes dessinées populaires : il est en effet le modèle du $\mathrm{P}^{\mathrm{r}}$ Sprtschk dans l'album Le Voyageur du Mésozoïque des Aventures de Spirou et de Fantasio (en 1957) et celui de Mik Ezdanitoff dans Vol 714 pour Sidney, album des Aventures de Tintin (en 1968). S’il est difficile de qualifier ces séries de "bandes dessinées pour adultes attardés », on ne peut nier le caractère science-fictionnel des Aventures de Spirou et Fantasio et de cet album de Tintin en particulier qui entre tout à fait en résonance avec la série télévisée qui nous occupe.

\section{Conclusion}

Finalement, ces séries sont loin de ressembler à ce que certains ont nommé la paléo-télévision. Textes réflexifs, cassant à plusieurs reprises le quatrième mur, tournés vers le téléspectateur, inscrits dans la familiarité du public, jouant avec lui, le laissant construire le sens du texte, ils semblent bien plutôt manifester des qualités que la doxa attribue de manière générale à une télévision plus tardive, comme si ce média avait dû passer par une enfance naïve avant de commencer à prendre conscience de lui-même, sans tirer parti de l'évolution des médias et des usages qui l'ont précédé. Si l'on relit la conclusion de l'article de Jean-Marie Schaeffer sur la réflexivité où il reprend les fondements de la théorie spéculative de l'art et de la poésie romantique, il semble bien que les exemples que constituent ces trois séries et feuilletons s'inscrivent dans une communauté de pratiques :

La conception actuelle de la "réflexivité" [...] engage une conception spécifique de la création artistique ou du moins de ce qui est censé importer dans cette création: la conscience réflexive de l'artifice plutôt que l'immersion dans un univers mimétique plausible, la problématisation de la tradition littéraire plutôt que la relation à la réalité extralittéraire, la complexité sémiotique plutôt que la richesse des univers modélisés, le paradoxe et la mise à nu des conventions plutôt que la reconduction des schèmes narratifs établis... (27)

Souligner dans le texte de la fiction que l'on regarde une série télévisée (comme le fait La Brigade des maléfices), dessiner l'évolution du médium par lequel on s'exprime (Le Voyageur des siècles pouvant être vu de cette façon, de même que certaines remarques d'Aux frontières du possible), inscrire dans le texte la trace de l'auteur (particulièrement dans $A u x$ frontières du possible, mais aussi par le dispositif du cameo), voici quelques-unes des pistes suivies ici. Si certaines de ces pratiques sont, par leur caractère explicite, en un sens transparentes, d'autres sont plus cryptiques, nécessitant soit des connaissances des conditions de production du texte (comme la référence à Jacques Bergier et la bande dessinée), soit un savoir sur les coulisses — au sens large — de la télévision. Dans tous les cas, elles nécessitent une capacité critique du téléspectateur, que les chercheurs, si l'on en suit les analyses d'Elihu Katz et de Tamar Liebes sur Dallas (1992, p. 125-126), ont commencé à attribuer au public au début des années 1980. Or, l'existence même de dispositifs jouant sur ces capacités critiques et sur 
ces savoirs dans des programmes de 1971 présuppose non seulement des créateurs capables de les mobiliser, mais aussi des téléspectateurs capables de les comprendre, sans quoi le risque d'échec est très grand. Il serait cependant hâtif de généraliser et de faire des séries de l'ORTF des équivalents des séries américaines actuelles qui se plaisent à multiplier les dispositifs réflexifs ${ }^{28}$. Le statut générique des programmes étudiés ici n'est sans doute pas étranger au constat que nous formulons. Denis Mellier explicite les affinités particulières de la réflexivité et de la spécularité avec deux genres transmédiatiques, le fantastique ${ }^{29}$ et le policier ${ }^{30}$ - tous deux représentés dans notre corpus - , et avec celui du thriller, qui partage avec celui de la série télévisée et du feuilleton une caractérisation populaire et une forte codification :

Si la portée réflexive est consubstantielle au thriller, c'est parce que, à mesure que mon expérience et ma mémoire de cinéma me font plonger dans la casuistique des fictions et la variation des dispositifs, me faisant goûter la reconnaissance et le plaisir des retrouvailles, le thriller n'agit qu'en m'exhibant le jeu des artifices qui déterminent ces conditions nécessaires à la feintise, jeu du make-believe, du faire comme si... Alors, à mesure que je m'absorbe dans la puissance de captation, la performance s'affiche, les symboles de ce que je fais dans la salle sombre, de ce que je suis venu chercher, se donnent à voir à l'écran, m'offrant au creux de mon plaisir la conscience que j'en ai. (Mellier 22)

Il serait évidemment faux de généraliser à la fois cette étude et ce que l'on peut inférer des jeux sur la codification formelle à partir des propos de Mellier et de présupposer une réflexivité dans toutes les séries et tous les feuilletons français des années $1970^{31}$ : la dimension fantastique joue, à notre avis, un rôle éminemment grand dans les cas que nous étudions. Mais ils permettent de relativiser certains propos et ouvrent une interrogation sur les modalités d'existence de cette réflexivité relativement

\footnotetext{
${ }^{28}$ Pour avoir un aperçu de cette réflexivité dans les séries américaines à partir des années 1990, on pourra se reporter à Barthes (2010 : 335-339; 492-500).

${ }^{29}$ « Si la portée réflexive s'impose dans la fiction fantastique, c'est parce qu'il n'y a de fantastique qu'à partir d'une interrogation sur les limites de l'apparition, sur les manières de faire tenir la chose dans la langue, la narration ou l'image, de l'accréditer dans une forme qui, pour le meilleur ou pour le pire, l'effroi ou le grotesque, la panique ou le rire libérateur, ne peut manquer de l'objectiver. » (Mellier 22)

30 «Si la portée réflexive est inhérente à la forme policière, c'est parce que toute entreprise policière, tout soupçon sur la culpabilité d'autrui, figure la condition de l'interprétant spectateur ou lecteur - doublée sur l'écran par la figure dominante de l'enquêteur, et que de façon symétrique toute élaboration criminelle figure un imaginaire retourné de la création, assimilant le criminel à l'auteur, ou les rituels pathologiques de mise en scène à ceux du cinéaste lui-même disposant figures et corps dans l'espace théâtralisé de son action. » (Mellier 22)

${ }^{31}$ Les faits, en effet, démentent ceux qui émettraient cette hypothèse : des séries plus purement policières de cette époque, comme Karatekas \& Co ou Les Nouvelles Aventures de $V i d o c q$, sont beaucoup moins réflexives, voire pas du tout,
} 
précoce alors que, d'après les chercheurs, la télévision américaine contemporaine de ces programmes ne s'est pas encore réellement ouvert cette perspective ${ }^{32}$.

${ }^{32}$ La Quality TV, dont l'on date habituellement le début de la fin des années 1970 et du début des années 1980, a plusieurs caractères similaires aux phénomènes que nous avons étudiés cet article: "Quality TV tends to be literary and writer-based. The writing is usually more complex than in other types of programming» et "Quality TV is self-conscious. Oblique allusions are made to both high and popular culture, but mostly to TV itself. [...] Both the classier cultural references and the sly, knowing jabs at TV serve to distance these programs for the stigmatized medium and to announce that they are superior to the typical trash available on television. " (Thompson, 1997: 15) 


\section{Bibliographie}

Barthes, R. "Il n'existe aucun discours qui ne soit une fiction", La Quinzaine littéraire, repris dans Barthes, R. (2002), CEuvres complètes tome IV : Livres, textes, entretiens 1972-1976, Paris: Seuil, (1976) : 937-939.

Barthes, S. Du «temps de cervean disponible »?, Thèse de doctorat sous la direction de Georges Molinié soutenue le 13 février 2010 à l'Université Paris-Sorbonne. Web. http://www.esorbonne.fr/theses/2010pa040011.

Barthes, S. «Le Retour du primetime. Une histoire de la programmation des séries télévisées étrangères à la télévision française », Communication \& Langages 174 (2012) : 123-145.

Baudou, J. \& J.-J. Schléret. Merveilleux, Fantastique et Science Fiction à la télévision française, Paris : Huitième Art, 1995.

Bourdon, J. «L'archaïque et le postmoderne », in Bourdon, J. et Jost, Fr. (dir.), Penser la télévision, Paris : Nathan (1998) : 15-28.

Casetti, F. et R. Odin. «De la paléo- à la néo-télévision », Communications 51 (1990) : 9-26.

Eco, U. La Guerre du faux, Paris : Grasset, 1985.

Ferré, M. «Un fantastique souriant », La Provence, 25 août 1971.

Jost, F., Introduction à l'analyse de la télévision, Paris : Ellipses, 1999.

Katz, E. \& T. Liebes. "Six interprétations de la série Dallas », Hermès 11-12 (1992) : 125-144.

Lagane, B. "Une si fantastique télévision française », Soap \#1, Paris : Éditions Libellus (2014) : 46-49.

Mellier, D. Les Écrans meurtriers. Essais sur les scènes spéculaires du thriller, Liège : Éditions du Cefal, 2002.

Michel, H. Les Grandes Dates de la télévision française, Paris : PUF, «Que saisje ?», 1995.

Piemme, J.-M. La Propagande inavouée, Paris : 10/18, 1975.

Schaeffer, J.-M. «Esthétique spéculative et hypothèses sur la réflexivité en art », in Bessière J. \& Schmeling, M. (dir.), Littérature, modernité, réflexivité, Paris : Honoré Champion (2002) : 15-27.

Spies, V. La Télévision au miroir. Théorie, histoire et analyse des émissions réflexives, Paris : L'Harmattan, 2004.

Spies, V. «De l'énonciation à la réflexivité : quand la télévision se prend pour objet», Semen 26, 2008 consulté en ligne le 5 mars http://semen.revues.org/8458.

Thompson, R. J. Television's Second Golden Age, Syracuse, New York: Syracuse University Press, 1997. 